Comment. Math. Helv. 72 (1997) 605-617

(C) 1997 Birkhäuser Verlag, Basel

$0010-2571 / 97 / 040605-13 \$ 1.50+0.20 / 0$

Commentarii Mathematici Helvetici

\title{
Cohen-Macaulay coordinate rings of blowup schemes
}

\author{
S. Dale Cutkosky and Jürgen Herzog*
}

\begin{abstract}
Suppose that $Y$ is a projective $k$-scheme with Cohen-Macaulay coordinate ring $S$. Let $I \subset S$ be a homogeneous ideal of $S$. I can be blown up to produce a projective $k$-scheme $X$ which birationally dominates $Y$. Let $I_{c}$ be the degree $c$ part of $I$. Then $k\left[I_{c}\right]$ is a coordinate ring of a projective embedding of $X$ for all $c$ sufficiently large. This paper considers the question of when there exists a constant $f$ such that $k\left[\left(I^{e}\right)_{c}\right]$ is Cohen-Macaulay for $c \geq e f$. A very general result is proved, giving a simple criterion for a linear bound of this type. As a consequence, local complete intersections have this property, as well as many other ideals.
\end{abstract}

Mathematics Subject Classification (1991). 14M05, $13 \mathrm{H} 10$.

Keywords. Cohen-Macaulay, coordinate ring.

\section{Introduction}

Suppose that $Y$ is a projective $k$-scheme with Cohen-Macaulay coordinate ring $S$. Let $I \subset S$ be a homogeneous ideal of $S$. Then I can be blown up to produce a projective $k$-scheme $X$ which birationally dominates $Y$. Let $I_{c}$ be the degree $c$ part of $I$. Then $k\left[I_{c}\right]$ is a coordinate ring of a projective embedding of $X$ for all $c$ sufficiently large. In general, $k\left[I_{c}\right]$ is not Cohen-Macaulay even when $X$ is CohenMacaulay (a simple example is given in Section 1). Recently, [3], [5], [6], [13] have given criteria for $k\left[I_{c}\right]$ to be Cohen-Macaulay in many important situations.

Powers $I^{e}$ of $I$ blow up to the same scheme $X$, and the rings $k\left[\left(I^{e}\right)_{c}\right]$ for $c \gg e>0$ are coordinate rings of projective embeddings of $X$.

In Theorem $4.6[3]$ an explicit necessary and sufficient linear bound in $c$ and $e$ is given for $k\left[\left(I^{e}\right)_{c}\right]$ to be Cohen-Macaulay, when $S$ is a polynomial ring of dimension $n$ and $I$ is a complete intersection in $S$. Suppose that the complete intersection ideal $I$ is minimally generated by forms of degree $d_{1}, \ldots, d_{r}$. Assume that $c \geq e d+1, d=\max \left\{d_{j} \mid j=1, \ldots, r\right\}$. Then $k\left[\left(I^{e}\right)_{c}\right]$ is a Cohen-Macaulay ring if and only if $c>\sum_{j=1}^{r} d_{j}+(e-1) d-n$.

This leads to the question of when there exists a constant $f$ such that $k\left[\left(I^{e}\right)_{c}\right]$

* First author partially supported by NSF. 
is Cohen-Macaulay for $c \geq e f$. In other words, when is there a linear bound on $c$ and $e$ ensuring that $k\left[\left(I^{e}\right)_{c}\right]$ is Cohen-Macaulay?

For instance, it is natural to expect that ideals $I$ that are local complete intersections (that is, $I S_{p}$ is a complete intersection if $p$ is not the irrelevant ideal of $S$ ) will have this property. The Kodaira Vanishing Theorem suggests that there should be a linear bound ensuring that $k\left[\left(I^{e}\right)_{c}\right]$ is Cohen-Macaulay, at least when a regular ideal is blown up in a nonsingular projective variety of characteristic zero.

In this paper, we prove a very general result (Theorem 4.1) giving a simple criterion for a linear bound of this type. As a consequence, we show (Corollary 4.2) that local complete intersections have this property, as well as many other ideals (Corollaries 4.3 and 4.4).

\section{Coordinate rings of a blowup}

Throughout this paper we will have the following assumptions. Let $k$ be a field, $S$ a noetherian graded $k$-algebra which is generated in degree 1 with graded maximal ideal $M$. Then $S$ has a presentation $S=k\left[x_{0}, x_{1}, \ldots, x_{n}\right] / K$, where each $x_{i}$ is homogeneous of degree 1 . Let $\beta=\operatorname{dim}(S), \bar{n}=\beta-1, I \subset S$ be a homogeneous ideal, and let $\tilde{I}$ be the sheaf associated to $I$ in $Y=\operatorname{Proj}(S)$. Let $X=\operatorname{Proj}\left(\bigoplus \tilde{I}^{n}\right)$ be the blowup of $\tilde{I}$, with natural map $\pi: X \rightarrow Y$. $I_{c}$ will denote the $c$-graded part of $I$.

Lemma 1.1. Suppose that $I$ is generated in degree $\leq d$. Then

(1) $\left(I_{c}\right) \cdot \mathcal{O}_{X}=\tilde{I}(c) \cdot \mathcal{O}_{X}$ for $c \geq d$.

(2) $I_{c}$ is a very ample subspace of $\Gamma\left(X, \tilde{I}(c) \cdot \mathcal{O}_{X}\right)$ for $c \geq d+1$.

Proof. Let $I$ be generated by $G_{1}, \ldots, G_{m}$ where the $G_{i}$ are homogeneous of degree $d_{i} \leq d$. Let

$$
R_{i j}=\left(k\left[\frac{x_{0}}{x_{i}}, \ldots, \frac{x_{n}}{x_{i}}\right] / K_{i}\right)\left[\frac{G_{1} x_{i}^{d_{j}-d_{1}}}{G_{j}}, \ldots, \frac{G_{m} x_{i}^{d_{j}-d_{m}}}{G_{j}}\right],
$$

$U_{i j}=\operatorname{Spec}\left(R_{i j}\right) .\left\{U_{i j}: 0 \leq i \leq n, 1 \leq j \leq m\right\}$ is an affine cover of $X . \Gamma\left(U_{i j}, \tilde{I}(c)\right.$. $\left.\mathcal{O}_{X}\right)=G_{j} x_{i}^{c-d_{j}} R_{i j}$.

Since $I_{c} \subset \Gamma\left(X, \tilde{I}(c) \cdot \mathcal{O}_{X}\right)$ and $G_{j} x_{i}^{c-d_{j}} \in I_{c}$ for $1 \leq j \leq m$ whenever $c \geq d$, we have (1).

To establish (2) we will use the criteria of Proposition II.7.2 [7]. Suppose that $c \geq d+1$. By (1), $I_{c}$ gives a morphism of $X$. $I_{c}$ is generated over $k$ by $\left\{G_{j} x_{0}^{l_{0}} x_{1}^{l_{1}} \cdots x_{n}^{l_{n}}: d_{j}+l_{0}+l_{1}+\cdots+l_{n}=c\right\}$. Suppose that $s=G_{j} x_{0}^{l_{0}} x_{1}^{l_{1}} \cdots x_{n}^{l_{n}}$ is one of these generators. Then some $l_{i}>0$, and $X_{s} \subset U_{i j}$.

$$
s=G_{j} x_{0}^{l_{0}} x_{1}^{l_{1}} \cdots x_{n}^{l_{n}}=G_{j} x_{i}^{c-d_{j}}\left(\frac{x_{0}}{x_{i}}\right)^{l_{0}} \cdots\left(\frac{x_{n}}{x_{i}}\right)^{l_{n}}
$$


so that $X_{s}=\operatorname{Spec}(A)$ where

$$
A=R_{i j}\left[\left(\frac{x_{i}}{x_{0}}\right)^{l_{0}} \cdots\left(\frac{x_{i}}{x_{n}}\right)^{l_{n}}\right] .
$$

We have

$$
\begin{gathered}
\frac{G_{j} x_{i}^{c-d_{j}}}{G_{j} x_{0}^{l_{0}} \cdots x_{n}^{l_{n}}}=\left(\frac{x_{i}}{x_{0}}\right)^{l_{0}} \cdots\left(\frac{x_{i}}{x_{n}}\right)^{l_{n}} \\
\frac{G_{j} x_{0}^{l_{0}} \cdots x_{t}^{l_{t}+1} \cdots x_{i}^{l_{i}-1} \cdots x_{n}^{l_{n}}}{G_{j} x_{0}^{l_{0}} \cdots x_{n}^{l_{n}}}=\frac{x_{t}}{x_{i}} \\
\frac{G_{t} x_{i}^{c-d_{t}}}{G_{j} x_{0}^{l_{0}} \cdots x_{n}^{l_{n}}} \cdot\left(\frac{G_{j} x_{0}^{l_{0}+1} \cdots x_{i}^{l_{i}-1} \cdots x_{n}^{l_{n}}}{G_{j} x_{0}^{l_{0}} \cdots x_{n}^{l_{n}}}\right)^{l_{0}} \cdots\left(\frac{G_{j} x_{0}^{l_{0}} \cdots x_{i}^{l_{i}-1} \cdots x_{n}^{l_{n}+1}}{G_{j} x_{0}^{l_{0}} \cdots x_{n}^{l_{n}}}\right)^{l_{n}} \\
=\frac{G_{t} x_{i}^{d_{j}-d_{t}}}{G_{j}}
\end{gathered}
$$

generate $A$ as a $k$-algebra.

Let $\mathcal{L}=\tilde{I} \cdot \mathcal{O}_{X}, \mathcal{M}=\pi^{*} \mathcal{O}_{Y}(1)$, so that $\left(I^{e}\right)_{c} \cdot \mathcal{O}_{X}=\mathcal{L}^{e} \otimes \mathcal{M}^{c}$ for $e>0$ and $c \geq d e$, and $X \cong \operatorname{Proj}\left(k\left[\left(I^{e}\right)_{c}\right]\right)$ for $c \geq d e+1$. In Lemma 1.2 we state the usual exact sequences relating local cohomology and global cohomology (cf. A4.1 [4]).

Lemma 1.2. Suppose that $I$ is generated in degree $\leq d, e>0$ and $c \geq d e+1$. Let $A=k\left[\left(I^{e}\right)_{c}\right]$, with graded maximal ideal $m$. There are exact sequences

$$
0 \rightarrow H_{m}^{0}(A) \rightarrow A \rightarrow \bigoplus_{s \in \mathbf{Z}} \Gamma\left(X, \mathcal{L}^{s e} \otimes \mathcal{M}^{s c}\right) \rightarrow H_{m}^{1}(A) \rightarrow 0
$$

and isomorphisms

$$
H_{m}^{i+1}(A) \cong \bigoplus_{s \in \mathbf{Z}} H^{i}\left(X, \mathcal{L}^{s e} \otimes \mathcal{M}^{s c}\right)
$$

for $i \geq 1$.

Let $I^{\star}$ denote the intersection of the primary components which are not $M$ primary of an irredundant primary decomposition of $I$.

Lemma 1.3. There exists a positive integer $f$ such that $\left(I^{a}\right)_{b}=\left(I^{a}\right)_{b}^{\star}$ for all $a, b$ with $b \geq f a$.

Proof. This is immediate from the Main Theorem of Swanson [14] (cf. also Theorem $1.5[10]$ ), which states that there exists an integer $f$ such that $I^{a}$ has an irredundant primary decomposition $I^{a}=q_{1} \cap \cdots \cap q_{s}$ with $\left(\sqrt{q_{i}}\right)^{a f} \subset q_{i}$ for all $i$. $\square$ 
Lemma 1.4. Suppose that no associated prime of $S$ contains $I$, $\operatorname{depth}_{M}(S) \geq 2$ and $\pi_{*}\left(\tilde{I}^{e} \cdot \mathcal{O}_{X}\right)=\tilde{I}^{e}$ for all $e \geq 0$. Then there exists a positive integer $f$ such that, with the notation of Lemma 1.2, $H_{m}^{0}(A)=0$ and $H_{m}^{1}(A)=0$ whenever $e>0$ and $c \geq e f$.

Proof. Suppose $I$ is generated in degree $\leq d$. By Lemma 1.3 there exists an integer $f^{\prime}$ such that $\left(I^{t}\right)_{s}=\left(I^{t}\right)_{s}^{\star}$ for $s \geq f^{\prime} t$. Set $f=\max \left(f^{\prime}, d+1\right)$.

By consideration of the natural inclusion $A \subset S[I t]$, we have $H_{M}^{0}(A)=0$ since $H_{I}^{0}(S)=0 . H^{0}\left(Y, \mathcal{O}_{Y}(s)\right)=H_{M}^{1}(S)_{s}=0$ for all $s<0$ since $\operatorname{depth}_{M}(S) \geq 2$. From the inclusions

$$
\Gamma\left(X, \mathcal{L}^{s e} \otimes \mathcal{M}^{s c}\right) \hookrightarrow \Gamma\left(X, \mathcal{M}^{s c}\right)=\Gamma\left(Y,\left(\pi_{*} \mathcal{O}_{X}\right) \otimes \mathcal{O}_{Y}(s c)\right)=\Gamma\left(Y, \mathcal{O}_{Y}(s c)\right)
$$

where the first equality is by the projection formula (cf. Exercise II.5.1 [7]), we get $\Gamma\left(X, \mathcal{L}^{s e} \otimes \mathcal{M}^{s c}\right)=0$ for $s<0$.

Let $\Delta$ be the $(c, e)$ diagonal of $\mathbf{Z}^{2}([3])$. For $c \geq e d$, we have $k\left[\left(I^{e}\right)_{c}\right]=S[I t]_{\Delta}$, as in Lemma 1.2 of [3].

$$
\begin{aligned}
\bigoplus_{s \geq 0} H^{0}\left(X, \mathcal{L}^{s e} \otimes \mathcal{M}^{s c}\right) & =\bigoplus_{s \geq 0} H^{0}\left(Y, \pi_{*}\left(\mathcal{L}^{s e}\right) \otimes \mathcal{O}_{Y}(s c)\right) \\
& =\bigoplus_{s \in \mathbf{Z}} H^{0}\left(Y, \tilde{I}^{s e}(s c)\right) \\
& =(\Gamma(\operatorname{Spec}(S)-M, \tilde{I}))_{\Delta} \\
& =\left(\bigoplus_{t \geq 0}\left(I^{t}\right)^{\star}\right)_{\Delta}=S[I t]_{\Delta} .
\end{aligned}
$$

Now Lemma 1.2 implies $H_{m}^{1}(A)=0$.

The condition of the existence of a Cohen-Macaulay coordinate ring is somewhat delicate, as shown by the following simple example of a Cohen-Macaulay scheme obtained by blowing up an ideal sheaf on a scheme with a Cohen-Macaulay coordinate ring, which does not have a Cohen-Macaulay coordinate ring. Let $T$ be a nonsingular "irregular" projective surface $\left(H^{1}\left(T, \mathcal{O}_{T}\right) \neq 0\right)$. Let $\pi: T \rightarrow U$ be a birational projection onto a hypersurface in $\mathbf{P}^{3} . \pi$ is the blowup of an ideal sheaf on $U$. The coordinate ring of $U$ is Cohen-Macaulay, and certainly $T$ is Cohen-Macaulay, but no coordinate ring of $T$ can be Cohen-Macaulay since $T$ is irregular.

However, we can give a simple proof of the existence of a linear bound ensuring that $k\left[\left(I^{e}\right)_{c}\right]$ is Cohen-Macaulay when $k$ has characteristic zero, $S$ is CohenMacaulay, $Y$ is nonsingular, $I$ is equidimensional and $\operatorname{Proj}(S / I)$ is nonsingular. The proof has three ingredients:

(1) The Kodaira Vanishing Theorem. 
(2) In this situation (everything nonsingular) $R^{i} \pi_{*} \mathcal{O}_{X}=0$ for $i>0$ and $\pi_{*} \mathcal{O}_{X}=\mathcal{O}_{Y}$ (cf. Proposition $10.2[11]$ ).

(3) Lemma 1.4.

If $\mathcal{N}$ is an ample invertible sheaf on a smooth projective variety $Z$ of characteristic zero and dimension $t$, with dualizing sheaf $\omega_{Z}$, then Kodaira Vanishing states that $H^{i}\left(Z, \mathcal{N} \otimes \omega_{Z}\right)=0$ for $i>0$. The Serre-dual form of Kodaira Vanishing is $H^{i}\left(Z, \mathcal{N}^{-1}\right)=0$ for $i<t$.

Proposition 1.5. Suppose that $k$ has characteristic zero, $S$ is Cohen-Macaulay, $Y$ is nonsingular, $I$ is equidimensional and $\operatorname{Proj}(S / I)$ is nonsingular. Then there exists a positive integer $f$ such that

$$
H^{i}\left(X, \mathcal{L}^{s e} \otimes \mathcal{M}^{s c}\right)=0
$$

for all $s \in \mathbf{Z}$ if $0<i<\bar{n}, c \geq e f, e>0$.

Proof. Suppose that $I$ has height $r$, and is generated in degree $<d$. By Lemma 1.1, $\mathcal{L}^{a} \otimes \mathcal{M}^{b}$ is very ample if $b>a d$. We immediately get $H^{i}\left(X, \mathcal{L}^{s e} \otimes \mathcal{M}^{s c}\right)=0$ if $c>e d, s<0$ and $i<\bar{n}$, since $\mathcal{L}^{e} \otimes \mathcal{M}^{c}$ is then ample.

$H^{i}\left(X, \mathcal{O}_{X}\right)=0$ for $0<i$, by the Leray spectral sequence, since $R^{i} \pi_{*} \mathcal{O}_{X}=0$ for $i>0, \pi_{*} \mathcal{O}_{X}=\mathcal{O}_{Y}$ and $H^{j}\left(Y, \mathcal{O}_{Y}\right)=0$ for $0<j$ (since $S$ is Cohen-Macaulay).

Let $\omega_{Y}$ be a dualizing sheaf on $Y$. $\omega_{Y}^{-1}(g)$ is ample on $Y$ for some $g>0$. $\omega_{X}=\mathcal{L}^{1-r} \otimes \omega_{Y}$ is a dualizing sheaf on $X$.

$$
\mathcal{L}^{e} \otimes \mathcal{M}^{c} \cong \mathcal{L}^{e+r-1} \otimes \mathcal{M}^{c} \otimes \omega_{Y}^{-1} \otimes \omega_{X}
$$

$\mathcal{L}^{e+r-1} \otimes \mathcal{M}^{c} \otimes \omega_{Y}^{-1}$ is ample if $c>g+(e+r-1) d$, and then $H^{i}\left(X, \mathcal{L}^{s e} \otimes \mathcal{M}^{s c}\right)=0$ for $s>0$ and $i>0$.

Theorem 1.6. Suppose that $k$ has characteristic zero, $S$ is Cohen-Macaulay, $Y$ is nonsingular, $I$ is equidimensional and $\operatorname{Proj}(S / I)$ is nonsingular. Then there exists a positive integer $f$ such that $k\left[\left(I^{e}\right)_{c}\right]$ is Cohen-Macaulay whenever $e>0$ and $c \geq e f$.

Proof. The assumptions of Lemma 1.4 are satisfied by Proposition 10.2 [11] (or Example 2.3) and Proposition III.8.5 [7]. By Lemmas 1.2, 1.4 and Proposition 1.5 there exists a positive integer $f$ such that $k\left[\left(I^{e}\right)_{c}\right]$ has depth $\bar{n}+1$ at $m$ whenever $e>0$ and $c \geq e f$.

Unfortunately, Kodaira Vanishing fails in positive characteristic or if anything is not (almost) nonsingular. However, we obtain a very general result which is sufficient for this global part of the argument in Section 3. The second ingredient is local. We give a very simple argument to generalize this part in Section 2. 


\section{Local conditions}

Lemma 2.1. Suppose that $R$ is a local ring, essentially of finite type over a field $k$ and $J \subset R$ is an ideal. Let $W=\operatorname{Spec}(R), V=\operatorname{Proj}\left(\bigoplus_{n \geq 0} J^{n}\right), E=$ $\operatorname{Proj}\left(\bigoplus_{n \geq 0} J^{n} / J^{n+1}\right), \mathcal{L}=\tilde{J} \cdot \mathcal{O}_{V}$. Suppose that

$$
\Gamma\left(E, \mathcal{O}_{E}(m)\right)=J^{m} / J^{m+1} \quad \text { for } m \geq 0
$$

and

$$
H^{i}\left(E, \mathcal{O}_{E}(m)\right)=0 \quad \text { for } i>0 \text { and } m \geq 0 .
$$

Then $\Gamma\left(V, \mathcal{L}^{m}\right)=J^{m}$ if $m \geq 0$ and $H^{q}\left(V, \mathcal{L}^{m}\right)=0$ for $q>0$ and $m \geq 0$.

Proof. Note that $\mathcal{L}=\mathcal{O}_{V}(1)$ on $V$.

We have exact sequences:

$$
0 \rightarrow \mathcal{O}_{V}(m+1) \rightarrow \mathcal{O}_{V}(m) \rightarrow \mathcal{O}_{E}(m) \rightarrow 0
$$

for all integers $m$. Thus we have surjections

$$
H^{i}\left(V, \mathcal{O}_{V}(m+1)\right) \rightarrow H^{i}\left(V, \mathcal{O}_{V}(m)\right)
$$

for $i>0$ and $m \geq 0$. Since $\mathcal{O}_{V}(m)$ is ample, we have $H^{i}\left(V, \mathcal{O}_{V}(m)\right)=0$ for all $m \gg 0$, and $i>0$, so we have all of the desired vanishing. We also have exact sequences:

$$
0 \rightarrow \Gamma\left(V, \mathcal{O}_{V}(m+1)\right) \rightarrow \Gamma\left(V, \mathcal{O}_{V}(m)\right) \rightarrow J^{m} / J^{m+1} \rightarrow 0
$$

for $m \geq 0$. Since $R$ is a localization of a finitely generated $k$-algebra, $\Gamma\left(V, \mathcal{O}_{V}(m)\right)=$ $J^{m}$ for $m \gg 0$ (cf. Exercise II.5.9 of [7]). Thus it follows from (1) that $\Gamma\left(V, \mathcal{O}_{V}(m)\right)=J^{m}$ for all $m \geq 0$.

Lemma 2.2. Let notation be as in Lemma 2.1. Suppose that $V$ is CohenMacaulay. Let $\omega_{V}$ be a dualizing sheaf on $V$ and $\omega_{E}$ be a dualizing sheaf on $E$. Suppose that $H^{i}\left(E, \omega_{E}(m)\right)=0$ for $i>0$ and $m \geq 2$. Then $H^{q}\left(V, \omega_{V} \otimes \mathcal{L}^{m}\right)=0$ for $q>0$ and $m \geq 1$.

Proof. The ideal sheaf of $E$ is $I \cdot \mathcal{O}_{V} \cong \mathcal{O}_{V}(1)$. By "adjunction" (cf. Proposition 2.4 [1] or Theorem III 7.11 [7]) we have

$$
\omega_{E} \cong \omega_{V} \otimes \mathcal{O}_{E}(-1)
$$

Since $\omega_{V}$ is Cohen-Macaulay, we deduce from the exact sequence

$$
0 \rightarrow \mathcal{O}_{V}(1) \rightarrow \mathcal{O}_{V} \rightarrow \mathcal{O}_{E} \rightarrow 0
$$


exact sequences:

$$
0 \rightarrow \omega_{V}(m+1) \rightarrow \omega_{V}(m) \rightarrow \omega_{E}(m+1) \rightarrow 0
$$

for all integers $m$.

Thus we have surjections

$$
H^{i}\left(V, \omega_{V}(m+1)\right) \rightarrow H^{i}\left(V, \omega_{V}(m)\right)
$$

for $i>0$ and $m \geq 1$. Since $\mathcal{O}_{V}(m)$ is ample, we have $H^{i}\left(V, \omega_{V}(m)\right)=0$ for all $m \gg 0$ and $i>0$, so we have all of the desired vanishing.

Example 2.3. Suppose that $R$ is a Cohen-Macaulay local ring, essentially of finite type over a field $k$ and $J \subset R$ is an ideal generated by a regular sequence. Then the conclusions of Lemmas 2.1 and 2.2 hold.

Proof. Let $f_{1}, \ldots, f_{r}$ be a minimal set of generators of $I . V$ is Cohen-Macaulay, $E=\operatorname{Proj}\left(\bigoplus_{n \geq 0} J^{n} / J^{n+1}\right) \cong \mathbf{P}_{R / J}^{r-1}, \omega_{E} \cong\left(\omega_{W} / J \omega_{W}\right) \otimes_{k} \mathcal{O}_{E}(-r)$. Now the assumptions of Lemmas 2.1 and 2.2 follow from the cohomology of projective space and the isomorphisms

$$
\begin{aligned}
H^{i}\left(X, \mathcal{O}_{E}(m)\right) & \cong R / J \otimes_{k} H^{i}\left(\mathbf{P}_{k}^{r-1}, \mathcal{O}(m)\right) \\
H^{i}\left(E,\left(\omega_{W} / J \omega_{W}\right) \otimes_{k} \mathcal{O}_{E}(m)\right) & \cong\left(\omega_{W} / J \omega_{W}\right) \otimes_{k} H^{i}\left(\mathbf{P}_{k}^{r-1}, \mathcal{O}(m)\right)
\end{aligned}
$$

by the Künneth formula (cf. p. 77 of [12]).

Let

$$
T=\bigoplus_{n \geq 0} J^{n} / J^{n+1}
$$

be the associated graded ring of $J$, and let $N$ be the ideal of positive degree elements of $T$. Suppose that $T$ is Cohen-Macaulay. Then there is a canonical module $W_{T}$ of $T$ such that the sheaf associated to $W_{T}$ is a dualizing sheaf $\omega_{E}$ on $E$. The vanishing hypotheses of Lemmas 2.1 and 2.2 hold whenever

$$
H_{N}^{i}(T)_{c}=0
$$

for $i \geq 0$ and $c \geq 0$ and

$$
H_{N}^{i}\left(W_{T}\right)_{c}=0
$$

for $i \geq 2$ and $c \geq 2$.

An ideal $J$ in a $\operatorname{ring} R$ is called strongly Cohen-Macaulay if the Koszul homology modules of $I$ with respect to a generating set are Cohen-Macaulay. Let $\mu(J)$ denote the minimal number of generators of an ideal $J$.

Example 2.4. Suppose that $R$ is a Gorenstein local ring, essentially of finite type over a field $k$ and $J \subset R$ is a strongly Cohen-Macaulay ideal, with $\mu\left(J_{P}\right) \leq$ 
height $(P)$ for all primes $P$ containing $J$. Then the conclusions of Lemma 2.1 and 2.2 hold.

Proof. Suppose $J$ is of height $g$ generated by $n$ elements. Let $S=R\left[X_{1}, \ldots, X_{n}\right]$ be a polynomial ring over $R$. Let $H(J)$ denote the Koszul homology $H\left(f_{1}, \ldots, f_{n}, R\right)$ where $f_{1}, \ldots, f_{n}$ are generators of $J . W_{R / J}=\operatorname{Ext}^{g}(R / J, R) \cong H_{n-g}(J)$ is the last non-vanishing $H_{i}(J)$. The approximation complex $\mathcal{M}$ is

$$
0 \rightarrow H_{n-g}(J) \otimes S(-n+g) \rightarrow \cdots \rightarrow H_{1}(J) \otimes S(-1) \rightarrow H_{0}(J) \otimes S \rightarrow 0 .
$$

In [8], it is shown that $H^{0}(\mathcal{M})=\oplus J^{n} / J^{n+1}$. By Theorems 2.5 and $2.6[8] \mathcal{M}$ is acyclic and $\oplus J^{n} / J^{n+1}, \oplus J^{n}$ are Cohen-Macaulay.

Let $\bar{S}=R / J\left[X_{1}, \ldots, X_{n}\right]$, with canonical module

$$
W_{\bar{S}}=W_{R / J} \otimes S(-n)=H_{n-g}(J) \otimes S(-n) .
$$

$H_{N}^{i}(T)$ is dual to $\operatorname{Ext}_{\bar{S}}^{n-i}\left(T, W_{\bar{S}}\right)\left(\operatorname{cf}\right.$. Theorem 3.6.19 [2]). We have $\operatorname{Ext}_{\bar{S}}\left(T, W_{\bar{S}}\right)=$ 0 for $i \neq n-g$ since $T$ is a Cohen-Macaulay module of $\operatorname{dimension} \operatorname{dim}(R)$, and $\operatorname{dim}(\bar{S})-\operatorname{dim}(T)=n-g$. By (c) of Theorem 2.6 [8] we can realize $W_{T}$ as the cokernel of

$$
H_{1}(J) \otimes S(-g-1) \rightarrow H_{0}(J) \otimes S(-g)
$$

so that $W_{T} \cong T(-g)$.

From this we see that $W_{T}$ is supported in degree $g$, so that $H_{N}^{g}(T)_{j}=0$ for $j>-g$ and $H_{N}^{g}\left(W_{T}\right)_{j}=0$ for $j>0$.

By a Theorem of Huneke (Theorem 1.14 [9]), all ideals in the linkage class of a complete intersection in a Gorenstein local ring are strongly Cohen-Macaulay. For instance, codimension 2 perfect and Gorenstein codimension 3 ideals are strongly Cohen-Macaulay.

Remark 2.5. The conclusions of Example 2.4 are true when $R$ is not Gorenstein but only Cohen-Macaulay.

In this case $\mathcal{M}^{*}$ is acyclic with zeroth homology $W_{T}$, and we can use $\mathcal{M}$ and $\mathcal{M}^{*}$ to compute the desired vanishing.

\section{Global conditions}

We return to the notation and hypotheses of Section 1.

Proposition 3.1. Suppose that

$$
R^{j} \pi_{*}\left(\tilde{I}^{a} \cdot \mathcal{O}_{X}\right)=0 \quad \text { for } 1 \leq a \leq \bar{n}+1, \quad j>0 .
$$


Then there exists a positive integer $f$ such that

$$
H^{j}\left(X, \mathcal{L}^{a} \otimes \mathcal{M}^{b}\right)=0 \quad \text { for } j>0, a>0 \text { and } b \geq f a .
$$

Proof. After possibly tensoring with an extension field of $k$, we may suppose that $k$ is an infinite field. Suppose that $I$ is generated in degree $\leq d$. Set $d^{\prime}=d+1$. Let $D_{1}, \ldots, D_{\bar{n}}$ be general members of $\Gamma\left(X, \mathcal{L} \otimes \mathcal{M}^{d^{\prime}}\right)$. Let

$$
L_{i}=D_{1} \cap \cdots \cap D_{i}
$$

be the (scheme theoretic) intersection. $L_{i}$ has dimension $\bar{n}-i$. Set $L_{0}=X$.

We have short exact sequences

$$
\begin{aligned}
0 \rightarrow\left(\mathcal{L}^{a} \otimes \mathcal{M}^{b}\right) \otimes \mathcal{O}_{L_{i}} & \rightarrow\left(\mathcal{L}^{a+1} \otimes \mathcal{M}^{b+d^{\prime}}\right) \otimes \mathcal{O}_{L_{i}} \\
& \rightarrow\left(\mathcal{L}^{a+1} \otimes \mathcal{M}^{b+d^{\prime}}\right) \otimes \mathcal{O}_{L_{i+1}} \rightarrow 0
\end{aligned}
$$

for all integers $a$ and $b$ and $0 \leq i \leq \bar{n}-1$.

$$
R^{j} \pi_{*}\left(\mathcal{L}^{a} \otimes \mathcal{M}^{b}\right)=R^{j} \pi_{*}\left(\tilde{I}^{a} \cdot \mathcal{O}_{X}\right) \otimes \mathcal{O}_{Y}(b)=0
$$

for $j>0$ and $1 \leq a \leq \bar{n}+1$. From the Leray spectral sequence

$$
H^{i}\left(Y, R^{j} \pi_{*}\left(\mathcal{L}^{a} \otimes \mathcal{M}^{b}\right)\right) \Rightarrow H^{i+j}\left(X, \mathcal{L}^{a} \otimes \mathcal{M}^{b}\right)
$$

we have

$$
H^{j}\left(X, \mathcal{L}^{a} \otimes \mathcal{M}^{b}\right)=H^{j}\left(Y, \pi_{*}\left(\tilde{I}^{a} \cdot \mathcal{O}_{X}\right) \otimes \mathcal{O}_{Y}(b)\right) .
$$

There thus exists an integer $f \geq d^{\prime}$ such that

$$
H^{j}\left(X, \mathcal{L}^{a} \otimes \mathcal{M}^{b}\right)=0 \quad \text { for } \quad j>0,1 \leq a \leq \bar{n}+1 \text { and } b \geq f
$$

since $\pi_{*}\left(\tilde{I}^{a} \cdot \mathcal{O}_{X}\right)$ is coherent and $\mathcal{O}_{Y}(1)$ is ample on $Y$.

By (3) and induction applied to the long exact cohomology sequences associated to (2) we have

$$
H^{j}\left(X, \mathcal{L}^{a} \otimes \mathcal{M}^{b} \otimes \mathcal{O}_{L_{i}}\right)=0 \text { for } j>0, i+1 \leq a \leq \bar{n}+1 \text { and } b \geq f+i d^{\prime}
$$

The following inductive statement $(5) \Longrightarrow(6)$ can be established by induction using the exact sequences (2), (4) and the equality

$$
(a, b)=(a-i-1)\left(1, d^{\prime}\right)+\left(i+1, b-(a-i-1) d^{\prime}\right) .
$$

Note that if $a \geq i+1$ and $b \geq f+(a-1) d^{\prime}$ then $b-(a-i-1) d^{\prime} \geq f+i d^{\prime}$. 
Suppose that

$$
H^{j}\left(X, \mathcal{L}^{a} \otimes \mathcal{M}^{b} \otimes \mathcal{O}_{L_{i+1}}\right)=0 \text { for } j>0, i+2 \leq a \text { and } b \geq f+(a-1) d^{\prime}
$$

Then

$$
H^{j}\left(X, \mathcal{L}^{a} \otimes \mathcal{M}^{b} \otimes \mathcal{O}_{L_{i}}\right)=0 \text { for } j>0, i+1 \leq a \text { and } b \geq f+(a-1) d^{\prime} .
$$

$L_{\bar{n}}$ has dimension 0 , so that (6) is immediate for $i=\bar{n}$. Thus the proposition follows from descending induction on $i$ using the above statement $(5) \Longrightarrow(6)$.

Proposition 3.2. Suppose that $X$ is a Cohen-Macaulay scheme and

$$
R^{j} \pi_{*}\left(\omega_{X} \otimes \mathcal{L}^{t}\right)=0 \quad \text { for } 1 \leq t \leq \bar{n}+1, j>0
$$

Then there exists a positive integer $f$ such that

$$
H^{j}\left(X, \mathcal{L}^{-a} \otimes \mathcal{M}^{-b}\right)=0 \quad \text { for } j<\bar{n}, a>0 \text { and } b \geq f a .
$$

Proof. After possibly tensoring with an extension field of $k$, we may suppose that $k$ is an infinite field. Suppose that $I$ is generated in degree $\leq d$. Set $d^{\prime}=d+1$. Let $D_{1}, \ldots, D_{\bar{n}}$ be general members of $\Gamma\left(X, \mathcal{L} \otimes \mathcal{M}^{d^{\prime}}\right)$. Let

$$
L_{i}=D_{1} \cap \cdots \cap D_{i}
$$

be the (scheme theoretic) intersection. $L_{i}$ has dimension $\bar{n}-i$. Set $L_{0}=X$.

We have short exact sequences

$$
\begin{aligned}
0 \rightarrow\left(\mathcal{L}^{-a-1} \otimes \mathcal{M}^{-b-d^{\prime}}\right) \otimes \mathcal{O}_{L_{i}} & \rightarrow\left(\mathcal{L}^{-a} \otimes \mathcal{M}^{-b}\right) \otimes \mathcal{O}_{L_{i}} \\
& \rightarrow\left(\mathcal{L}^{-a} \otimes \mathcal{M}^{-b}\right) \otimes \mathcal{O}_{L_{i+1}} \rightarrow 0
\end{aligned}
$$

for all integers $a$ and $b$ and $0 \leq i \leq \bar{n}-1$.

By Serre-duality,

$$
\begin{aligned}
H^{j}\left(X, \mathcal{L}^{-a} \otimes \mathcal{M}^{-b}\right) & =H^{\bar{n}-j}\left(X, \omega_{X} \otimes \mathcal{L}^{a} \otimes \mathcal{M}^{b}\right) . \\
R^{j} \pi_{*}\left(\omega_{X} \otimes \mathcal{L}^{a} \otimes \mathcal{M}^{b}\right) & =R^{j} \pi_{*}\left(\omega_{X} \otimes \mathcal{L}^{a}\right) \otimes \mathcal{O}_{Y}(b)=0
\end{aligned}
$$

for $j>0$ and $1 \leq a \leq \bar{n}+1$. From the Leray spectral sequence, we hav

$$
H^{\bar{n}-j}\left(X, \omega_{X} \otimes \mathcal{L}^{a} \otimes \mathcal{M}^{b}\right)=H^{\bar{n}-j}\left(Y, \pi_{*}\left(\omega_{X} \otimes \mathcal{L}^{a}\right) \otimes \mathcal{O}_{y}(b)\right) .
$$

Hence there exists an integer $f \geq d^{\prime}$ such that

$$
H^{j}\left(X, \mathcal{L}^{-a} \otimes \mathcal{M}^{-b}\right)=0 \quad \text { for } \quad j<\bar{n}, 1 \leq a \leq \bar{n}+1 \text { and } b \geq f
$$


since $\pi_{*}\left(\omega_{X} \otimes \mathcal{L}^{a}\right)$ is coherent and $\mathcal{O}_{Y}(1)$ is ample on $Y$.

By (8) and induction applied to the long exact cohomology sequences associated to (7) we have

$$
H^{j}\left(X, \mathcal{L}^{-a} \otimes \mathcal{M}^{-b} \otimes \mathcal{O}_{L_{i}}\right)=0 \text { for } j<\bar{n}-i, 1 \leq a \leq \bar{n}+1-i \text { and } b \geq f .
$$

The following inductive statement $(10) \Longrightarrow(11)$ can be established by induction using the exact sequences (7), (9) and the equality

$$
(a, b)=(a-1)\left(1, d^{\prime}\right)+\left(1, b-(a-1) d^{\prime}\right) .
$$

Note that if $a \geq 1$ and $b \geq f+(a-1) d^{\prime}$ then $b-(a-1) d^{\prime} \geq f$.

Suppose that

$$
H^{j}\left(X, \mathcal{L}^{-a} \otimes \mathcal{M}^{-b} \otimes \mathcal{O}_{L_{i+1}}\right)=0 \text { for } j<\bar{n}-i-1,1 \leq a \text { and } b \geq f+(a-1) d^{\prime} .
$$

Then

$$
H^{j}\left(X, \mathcal{L}^{-a} \otimes \mathcal{M}^{-b} \otimes \mathcal{O}_{L_{i}}\right)=0 \text { for } j<\bar{n}-i, 1 \leq a \text { and } b \geq f+(a-1) d^{\prime} .
$$

(11) is immediate for $i=\bar{n}$. Thus the proposition follows from descending induction on $i$ using the above statement (10) $\Longrightarrow(11)$.

\section{Linear bounds for Cohen-Macaulay coordinate rings}

Let $k$ be a field, $S$ a noetherian graded $k$-algebra which is generated in degree 1, with graded maximal ideal $M$. Let $I \subset S$ be a homogeneous ideal, and let $\tilde{I}$ be the sheaf associated to $I$ in $Y=\operatorname{Proj}(S)$. Let $X=\operatorname{Proj}\left(\bigoplus \tilde{I}^{n}\right)$ be the blowup of $\tilde{I}$, with natural map $\pi: X \rightarrow Y$, and $\mathcal{O}_{X}(1)=\tilde{I} \cdot \mathcal{O}_{X}$. Let $\beta$ be the dimension of $S, \bar{n}=\beta-1$ be the dimension of $Y$.

Theorem 4.1. Suppose that $I$ is an ideal of height $>0, S$ is Cohen-Macaulay and $X$ is a Cohen-Macaulay scheme. Let

$$
E=\operatorname{Proj}\left(\bigoplus_{n \geq 0} \tilde{I}^{n} / \tilde{I}^{n+1}\right)
$$

with dualizing sheaf $\omega_{E}$. Suppose that

$$
\begin{aligned}
\pi_{*} \mathcal{O}_{E}(m) & =\tilde{I}^{m} / \tilde{I}^{m+1} \text { for } m \geq 0, \\
R^{i} \pi_{*} \mathcal{O}_{E}(m) & =0 \text { for } i>0 \text { and } m \geq 0 \text { and } \\
R^{i} \pi_{*} \omega_{E}(m) & =0 \text { for } i>0 \text { and } m \geq 2 .
\end{aligned}
$$


Then there exists a positive integer $f$ such that $k\left[\left(I^{e}\right)_{c}\right]$ is Cohen-Macaulay whenever $e>0$ and $c \geq e f$.

Proof. $R^{i} \pi_{*} \mathcal{O}_{X}=0$ for $i>0$ and $\pi_{*} \mathcal{O}_{X}=\mathcal{O}_{Y}$ by Lemma 2.1 (and Proposition III.8.5 [7]). $S$ is Cohen-Macaulay so that $H_{M}^{i}(S)=0$ for $i<\beta$ and $H^{i}\left(Y, \mathcal{O}_{Y}\right)=$ 0 for $0<i<\bar{n}$. Now by the Leray spectral sequence, $H^{i}\left(Y, R^{j} \pi_{*} \mathcal{O}_{X}\right) \Rightarrow$ $H^{i+j}\left(X, \mathcal{O}_{X}\right)$, we get $H^{i}\left(X, \mathcal{O}_{X}\right)=0$ for $0<i<\bar{n}$.

By Lemma 2.1 and Proposition 3.1 we have an $f$ such that $H^{j}\left(X, \mathcal{L}^{a} \otimes \mathcal{M}^{b}\right)=0$ for $j>0, a>0, b \geq f a$. By Lemma 2.2 and Proposition 3.2 there exists $f$ such that $H^{j}\left(X, \mathcal{L}^{-a} \otimes \overline{\mathcal{M}}^{-b}\right)=0$ for $j<\bar{n}, a>0, b \geq f a$.

Now the Theorem follows from Lemmas 1.4 and 1.2.

Corollary 4.2. Suppose that $S$ is Cohen-Macaulay, $I$ is an ideal of height $>0$ and $\tilde{I}$ is locally a complete intersection in $Y=\operatorname{Proj}(S)$. Then there exists a positive integer $f$ such that $k\left[\left(I^{e}\right)_{c}\right]$ is Cohen-Macaulay whenever $e>0$ and $c \geq e f$.

Proof. This is immediate from Example 2.3.

The following Corollary is now immediate from the comments following Example 2.3. By a canonical module $W_{T}$ we mean a canonical module whose associated sheaf is a dualizing sheaf of $\operatorname{Proj}(T) . I_{(P)}^{n}$ denoted the degree 0 elements of the localization $I_{P}^{n}$.

Corollary 4.3. Suppose that

(1) $S$ is Cohen-Macaulay.

(2) $I$ is an ideal of height $>0$.

(3) $\bigoplus_{n \geq 0} I_{(P)}^{n}$ and $T(P)=\bigoplus_{n \geq 0}\left(I^{n} / I^{n+1}\right)_{(P)}$ are Cohen-Macaulay for all $P \in \operatorname{Proj}(S)$.

(4) $H \frac{i}{P}(T(P))_{c}=0$ for $i \geq 0$ and $c \geq 0$ and $H \frac{i}{P}\left(W_{T(P)}\right)_{c}=0$ for $i \geq 2$ and $c \geq 2$ for all $P \in \operatorname{Proj}(S)$, where $W_{T(P)}$ is the canonical module of $T(P)$, $\bar{P}$ is the maximal ideal of $S_{(P)}$.

Then there exists a positive integer $f$ such that $k\left[\left(I^{e}\right)_{c}\right]$ is Cohen-Macaulay whenever $e>0$ and $c \geq e f$.

Corollary 4.4. Suppose that $S$ is Cohen-Macaulay, $I$ is an ideal of height $>0$ and $I_{(P)}$ is strongly Cohen-Macaulay with $\mu\left(I_{(P)}\right) \leq$ height $(P)$ for all primes $P \in Y$ containing $I$. Then there exists a positive integer $f$ such that $k\left[\left(I^{e}\right)_{c}\right]$ is Cohen-Macaulay whenever $e>0$ and $c \geq e f$.

Proof. The assumptions of Theorem 4.1 are satisfied by Example 2.4 and Remark 2.5. 


\section{References}

[1] A. Altman and S. Kleiman, Introduction to Grothendieck duality theory, Lecture Notes in Math. 146, Springer Verlag, 1970.

[2] W. Bruns and J. Herzog, Cohen-Macaulay rings, Cambridge Univ., 1993.

[3] A. Conca, J. Herzog, N. V. Trung and G. Valla, Diagonal subalgebras of bigraded algebras and embeddings of blow-ups of projective spaces, Amer. J. Math. 119 (1997), 859-901.

[4] D. Eisenbud, Commutative Algebra with a view toward algebraic geometry, Springer Verlag, 1995.

[5] A. Geramita, A. Gimigliano and B. Harbourne, Projectively normal but superabundant embeddings of rational surfaces in projective spaces, J. Algebra 169 (1994), 791-213.

[6] A. Geramita, A. Gimigliano and Y. Pitteloud, Graded Betti numbers of some embedded rational $n$-folds, Math. Annalen 301 (1995), 363-380.

[7] R. Hartshorne, Algebraic Geometry, Springer Verlag, 1977.

[8] J. Herzog, A. Simis and W. V. Vasconcelos, Approximation complexes of blowing-up rings, J. Alg. 74 (1982), 466-493.

[9] C. Huneke, Linkage and the Koszul homology of ideals, Amer. J. Math. 104 (1982), 1043-1062.

[10] D. Katz and S. McAdam, Two asymptotic functions, Comm. in Alg. 17 (1989), 1069-1091.

[11] H. Matsumura, Geometric structure of the cohomology rings in abstract algebraic geometry, Mem. Coll. Sci. Univ. Kyoto (A) 32 (1959), 33-84.

[12] D. Mumford, Annals of Math. Studies 59, Lectures on Curves on an algebraic surface, Princeton U. Press, 1966.

[13] A. Simis, N. V. Trung, and G. Valla, The diagonal subalgebra of a blow-up algebra. Preprint.

[14] I. Swanson, Powers of ideals: Primary decompositions, Artin-Rees Lemma and Regularity. Math. Annalen 307 (1997), 299-313.

\section{Jürgen Herzog}

FB 6 Mathematik und Informatik

Universität-GHS-Essen

Postfach 103764

D-45117 Essen

Germany

e-mail: mat300@uni-essen.de
S. Dale Cutkosky

Department of Mathematics

University of Missouri

Columbia, MO 65211

USA

e-mail: dale@cutkosky.math.missouri.edu

(Received: January 5, 1997) 